\title{
Interstrand disulfide crosslinking of DNA bases supports a double nucleotide unpairing mechanism for flap endonucleases
}

\author{
Amanda Beddows, ${ }^{a \star}{ }^{\ddagger}$ Nikesh Patel,${ }^{a, \ddagger}$ L David Finger, ${ }^{a}$ John M Atack, ${ }^{a, b}$ David M Williams ${ }^{a}$ and Jane A \\ $\operatorname{Grasby}^{a}$
}

Received (in $X X X, X X X)$ Xth $X X X X X X X X X 20 X X$, Accepted $X$ th $X X X X X X X X X 20 X X$

DOI: 10.1039/b000000x

Flap endonucleases (FENs) are proposed to select their target phosphate diester by unpairing the two terminal nucleotides of the reacting duplex. Interstrand disulfide crosslinks, introduced by oxidation of thiouracil and thioguanine bases, abolished the specificity of human FEN1 for hydrolysis one nucleotide into the 5 '-duplex.

Site-specific hydrolysis of the phosphate diester bonds of nucleic acids with aberrant structure is an essential step to the restoration of duplex DNA. Thus, phosphodiesterases that target nucleic acids by sensing unusual secondary and tertiary structure rather than sequence play critical roles in DNA replication, repair and recombination. For example, several protein-sequence related structure-sensing phosphodiesterases act as 5'-nucleases. This family of enzymes targets the 5'-region of DNA duplexes contained within more complex nucleic acid structures and catalyse hydrolyses one nucleotide into the double strand ${ }^{1}$.

Flap endonucleases (FENs), the prototypical member of the 5'nuclease family, are conserved throughout all domains of life. FENs catalyse the essential removal of 5'-single-stranded RNA or DNA extensions to duplexes, known as flaps. During discontinuous lagging-strand replication, 5'-flaps are formed during DNA polymerase catalysed extension of multiple RNA primers to create Okazaki fragments. Extension of each Okazaki fragment displaces the 5'-end (primer) of the previous fragment from the template strand. During a typical mammalian cell-cycle, FEN1 must remove the 5'-flaps (primers) of $\sim 50$ million Okazaki fragments to allow the completion of replication by ligation ${ }^{2}$.
Precise reaction site selection is critical to FEN1 function. Both the 5'-primer of one Okazaki fragment and newly synthesized DNA of the other are complementary to the template strand. Therefore, flaps created during replication can adopt a number of different interconvertible structures. The conformer specifically recognised by eukaryotic FEN1 proteins is a double flap (DF) with any length of 5'-flap (including none), but only a single nucleotide 3'-flap. Because the 3'-flap is complementary to the template, FEN1-catalysed hydrolysis one nucleotide into the 5'-duplex forms nicked DNA, a substrate for DNA ligase. However, the mechanism used by FEN1 and other 5'-nucleases to select only the first 5'-phosphate diester of the duplex for reaction and to discriminate against genome endangering hydrolyses of others has been challenging to unravel.

Like many phosphodiesterases, 5'-nucleases require $\mathrm{Mg}^{2+}$ cofactors. A typical metallonuclease has two active site divalent metal ions that inner-sphere coordinate the scissile phosphate diester, thereby acting as Lewis acid catalysts ${ }^{3}$. Reaction site specificity of FEN1 superfamily proteins was proposed to be explained by a double nucleotide unpairing (DNU) mechanism, which only allows the specific phosphate diester to access active site metal ions ${ }^{2,4-6}$ (Figure 1). This mechanism was initially inferred from X-ray structures of FEN proteins with substrate, where the duplex to undergo reaction was seen bound in front of, but not within the metal ion free active site (i.e., still basepaired) ${ }^{4}$. More recently, structures of human FEN1 (hFEN1) and another superfamily member hEXO1 bound to product DNAs provided support for this mechanism ${ }^{2,6}$. In these complexes, the
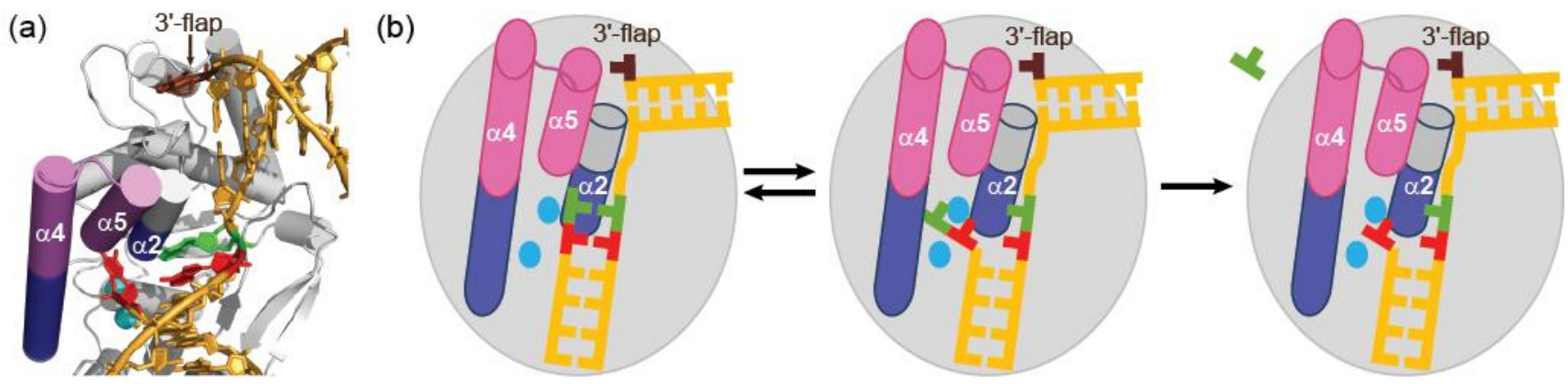

Figure 1. (a) hFEN1-product DNA complex (3Q8L) with the unpaired -1 nucleotide (nt) and its template strand partner in red. The hydrolysed phosphate monoester interacts with the two metal ions (cyan) in the active site. The template strand nt that lacks a base pair partner due to reaction is shown as green and the 3'-nt flap is brown. (b) Schematic of hFEN1 catalytic cycle coloured as in (a). After initial substrate binding in base-paired form (implied by this study) the reacting duplex must undergo double nucleotide unpairing (DNU) to allow the scissile phosphate diester to contact active site metal ions. The helical gateway (blue) is too narrow for duplex DNA to access active site metal ions without unpairing. 
hydrolysed 5'-phosphate monoester was directly coordinated to two active site metal ions; a conformation that required the terminal nucleotide (nt) of product to be unpaired (Figure 1a). Thus, it is supposed that the two terminal nts of substrate duplex must unpair for reaction to occur (Figure 1b).

To investigate a requirement for nucleotide unpairing in FEN reactions, we prepared DF substrates containing disulfide crosslinks at "base pairs" either side of the scissile phosphate diester bond (Figure 2a-b and Supplemental Figure S1). Dynamic simulations show that such crosslinks are tolerated in B-DNA ${ }^{10}$. To facilitate crosslinking, but mimic the preferred conformer of a biological hFEN1 substrate, the single nt 3'-flap and the $5 \mathrm{nt} 5$ 'flap were not complementary to the template strand. Like flaps formed during replication, such constructs undergo a specific hFEN1 catalysed reaction one nucleotide into the duplex portion of the 5'-flap strand ${ }^{7}$. Thus alongside 5'-monophosphorylated product, FEN catalysed reaction produces a single-stranded product with a 3'-hydroxyl group that is one nucleotide longer than the $5^{\prime}$-flap (here, this product is $\left.6 \mathrm{nt},\right)^{8}$. (a)<smiles></smiles>

(b) ${ }^{3}$ 5' Template

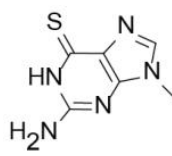
Denaturant 5' FAM 3'
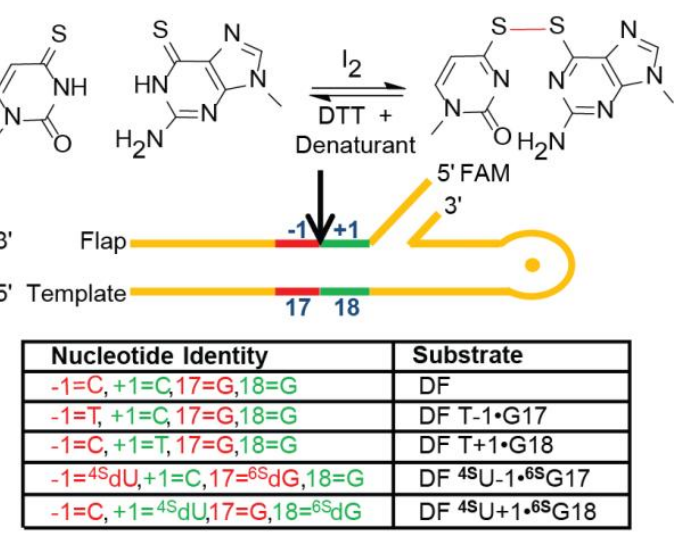

(c)

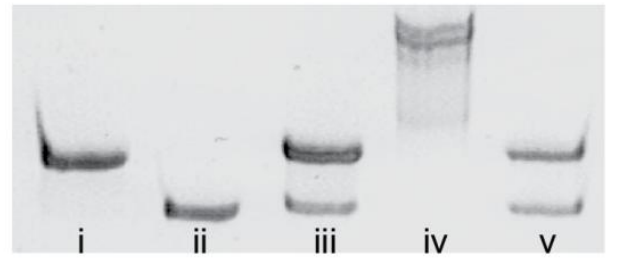

Figure 2. (a) Formation of the disulfide by $I_{2}$ oxidation. (b) Schematic of double flap substrate with 5-nt 5'-flap and a $1 \mathrm{nt} \mathrm{3'}$ 'flap; positions of substitution relative to reaction site (black arrow) are highlighted and tabulated for each substrate. (c) 20\% denaturing (8 M urea) PAGE gel visualised with toluidine blue [i] ${ }^{65} \mathrm{G} 17$, [ii] ${ }^{4 \mathrm{~S}} \mathrm{U}-1$, [iii] DF ${ }^{4 \mathrm{~S}} \mathrm{U}-1 \cdot{ }^{6 \mathrm{~S}} \mathrm{G} 17$ (no oxidation), [iv] oxidised and HPLC purified $\mathrm{DF}^{4 \mathrm{~S}} \mathrm{U}-1 \cdot{ }^{6 \mathrm{~S}} \mathrm{G} 17$ and $[\mathbf{v}]$ oxidised and purified $\mathrm{DF}{ }^{4 \mathrm{~S}} \mathrm{U}$ $1 .{ }^{6 \mathrm{~S}} \mathrm{G} 17$ treated with $10 \mathrm{mM}$ DTT in $50 \%$ formamide.

The double flap substrates were assembled from two oligodeoxyribonucleotides (ODNs), a 2'-deoxy-4-thiouridine $\left({ }^{4 S} \mathrm{U}\right)$ containing flap strand and a $2^{\prime}$-deoxy-6-thioguanosine $\left({ }^{6 \mathrm{~S}} \mathrm{G}\right)$ containing template strand, to form a non-Watson Crick ${ }^{4 S} \mathrm{U} \cdot{ }^{6 \mathrm{~S}} \mathrm{G}$ base pair (Figure 2a-b, Supplemental Figure S1 and Tables S1-2). ODNs containing the thionucleosides were assembled with commercially available $S$-cyanoethyl phosphoramidites (Supplemental Methods) with a 5'-fluorescein (FAM) label on flap strands to allow monitoring of the enzyme catalysed reaction. After HPLC purification, ODNs were characterised by mass spectrometry (Supplemental Table S1).
Disulfide formation was monitored by reversed phase HPLC under denaturing conditions $\left(60^{\circ} \mathrm{C}\right)$ (Supplemental Figure S2) and by denaturing polyacrylamide gel electrophoresis (PAGE) (Figure 2c). Upon annealing of flap and template strands, the thionucleosides did not spontaneously form disulfides, even under hFEN1 reaction conditions. In contrast, in model duplexes not supplied within the context of the DF structure, we did observe some spontaneous oxidation ${ }^{9}$. However, disulfide was formed within the DFs upon addition of $\mathrm{I}_{2}$ (10-20 equivalents with respect to concentration of annealed substrate $)^{10}$. No side reactions of the unmodified nucleic acid occurred during iodine treatment, but desulfurisation of the thionucleosides occurred in the absence of crosslinking partner and iodination (mainly di-) of the FAM moiety of flap strands also occurred under these conditions (Supplemental Table S1). Isolation of the crosslinked substrates, which eluted later than the individual flap and template strands, was readily achieved by HPLC.

The purified crosslinked substrates migrated more slowly than the constituent ODNs on denaturing PAGE (Figure 2c). The two banding pattern observed for the disulfide is characteristic of crosslinked flapped structures and has been attributed to different orientations of the denatured 5 '- and 3 '-extensions ${ }^{11}$. The disulfide was reduced by addition of dithiothreitol (DTT) in the presence of $50 \%$ formamide or $7 \mathrm{M}$ urea, but in the absence of denaturant was surprisingly resistant to reduction. The crosslinked substrate was stable under FEN reaction conditions (50 mM HEPES $\mathrm{pH}$ 7.5, $100 \mathrm{mM} \mathrm{KCl}, 8 \mathrm{mM} \mathrm{MgCl} 2,1 \mathrm{mM} \mathrm{DTT}$ ) for at least 6 hours. In subsequent enzyme experiments, all reactions gave identical products regardless of whether the reducing agent was present, although rates of reaction were approximately 2 -fold greater in the presence of DTT, presumably due to protection of the enzyme from oxidation.

The specificities of hFEN1-catalysed reactions were monitored by denaturing reversed phase HPLC equipped with a fluorescent detector, using tetrabutylammonium bromide buffers and a gradient of acetonitrile ${ }^{7}$ (Supplemental Methods). This ionpairing reagent allowed single nucleotide separation of short fluorescent ODNs using a DNA Sep column (Transgenomic). Diiodination of the FAM-label increased retention time; an effect more marked for shorter ODNs with the gradient used (Figure 3). The retention times of 5'-FAM flap products of hFEN1-catalysed reaction were compared to independently synthesized standards of potential reaction products. Enzyme reactions were stopped by addition of an equal volume of stop buffer containing $250 \mathrm{mM}$ EDTA, $8 \mathrm{M}$ urea and $1 \mathrm{mM}$ DTT (reducing conditions).

A non-thio T-1·G17 mismatch in the DF substrate produced the same reaction specificity (6 nt product) and similar rate of reaction to a fully Watson-Crick base paired substrate (Figure 3a and $b$ [iii] left). However, despite a postulated requirement for unpairing, introduction of a $\mathrm{T}+1 \cdot \mathrm{G} 18$ pair at the terminus of the reacting duplex slowed the hFEN1-catalysed reaction 30-fold (Figure 3a). Enzyme-catalysed reaction of $\mathrm{T}+1 \cdot \mathrm{G} 18$ produced both longer and shorter 5'-reaction products (5-7 mer) compared to DF T-1·G17, corresponding to reactions further into the duplex and within the single-stranded flap (Figure $3 b$ [iii] right). Thus, base pair stability at this position influences scissile phosphate selectivity. For both mismatched DF substrates, similar products having slightly longer retention times due to treatment with $I_{2}$ to 


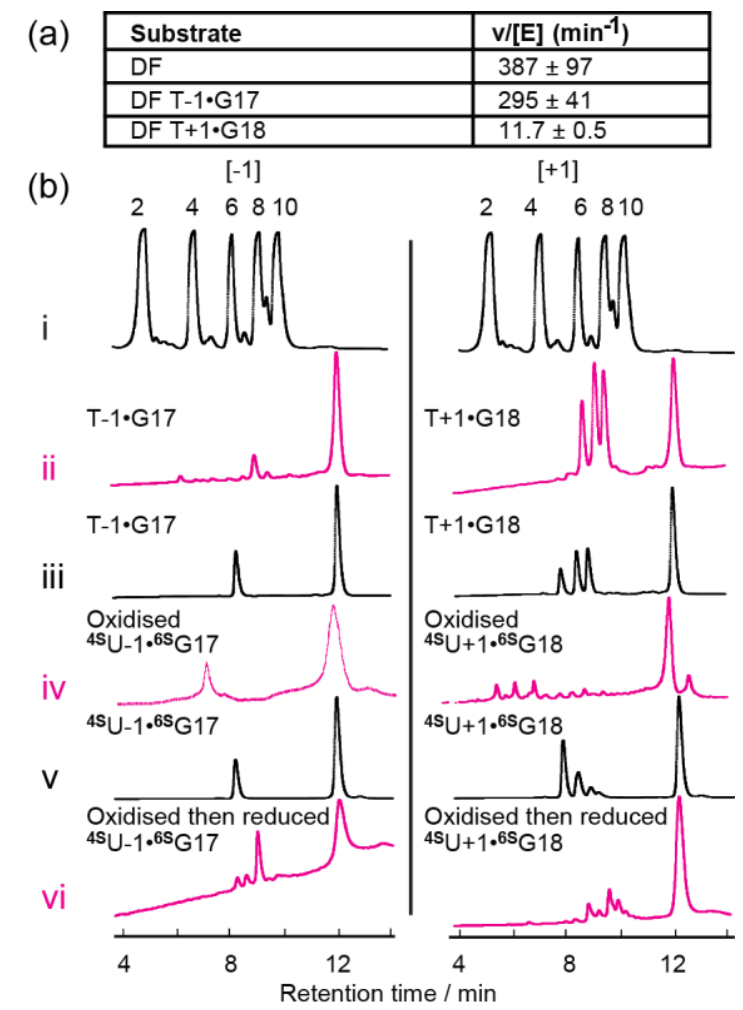

Figure 3. (a) Rates of hFEN1-catalysed reaction of DF (base paired), $\mathrm{DF} \mathrm{T}-1 \cdot \mathrm{G} 17$ (mismatch) and $\mathrm{DF} \mathrm{T}+1 \cdot \mathrm{G} 18$ (mismatch). Reactions were carried out at $37^{\circ} \mathrm{C}$ in $50 \mathrm{mM}$ HEPES pH 7.5, $100 \mathrm{mM} \mathrm{KCl}, 8$ $\mathrm{mM} \mathrm{MgCl} 2,1 \mathrm{mM}$ DTT, and $0.1 \mathrm{mg} / \mathrm{ml}$ BSA using $100 \mathrm{nM}$ substrate. Data are the result of three independent experiments. (b) HPLC traces of hFEN1-catalysed reactions of FAM (black) or iodoFAM (magenta) DF substrates $(100 \mathrm{nM})$ detected by fluorescence. Left modification or mismatches $-1 \bullet 17$, right $+1 \bullet 18$. [i] Standards length shown. [ii]-[vi] DF substrates as indicated. Peak at $\sim 12 \mathrm{~min}$ is starting material. Traces are of reactions conducted to show the size products of reaction, rather than determination of rate (Supplemental Methods).

give iodoFAM were observed (Figure 3b[ii]).

Modification of substrates to include ${ }^{4 \mathrm{~S}} \mathrm{U}$ at positions +1 or -1 , together with the corresponding introductions of ${ }^{6 S} \mathrm{G}$ in the template strands, produced hFEN1-reaction products similar to those seen with the corresponding non-thio-mismatched $\mathrm{T} \cdot \mathrm{G}$ FAM substrates (Figure $3 \mathrm{~b}[\mathrm{v} \quad c f$. iii]), indicating that the introduction of thio-nucleosides alone does not alter hFEN1 reaction specificity. However, when a disulfide crosslink was formed involving either of the thio "base pairs", hydrolysis within the duplex was completely prevented. Instead, much slower reactions requiring elevated enzyme concentrations produced products eluting earlier in the gradient, corresponding to hydrolysis within the single-stranded 5'-flap (Figure 3b[iv $c f$. ii]; $\sim 3$ mer). Furthermore, when disulfide substrates were pre-treated with DTT under denaturing conditions and then re-annealed, FEN reaction sites were very similar to those produced by the equivalent iodoFAM mismatched species (Figure $3 \mathrm{~b}$ [vi $c f$. ii]). Thus, at either side of the target phosphate diester, the characteristic hFEN1 reaction specificity is abolished by crosslinks designed to prevent DNU.

The disulfide DF substrates provide the first functional evidence for DNU of hFEN1 substrates. Other 5'-nucleases, whose activities span all major DNA metabolic pathways, catalyse reactions one nucleotide into a duplex of DNA bubbles
(XPG), four way duplex junctions (GEN1) and nicked or gapped DNAs (EXO1 and lower evolutionary FENs) ${ }^{1}$. These other 5'nuclease family members all conserve the hFEN1 active site, and therefore, are highly likely to also use DNU to achieve reaction specificity. Interestingly, the slowed rate of reaction and loss of specificity observed here when a non-Watson Crick $\mathrm{T} \cdot \mathrm{G}$ pair is present at the 5'-terminus of the reacting duplex implies a basepaired double strand is a preferred intermediate on the hFEN1 reaction pathway prior to unpairing (Figure 1b). For other superfamily members that do not recognize the 3'-flap bound by hFEN1 as a consequence of its replicative specialisation, the terminal Watson-Crick base pair of the reacting duplex will be the initial recognition feature that ultimately defines the site of reaction.

The crosslinked DF substrates also illustrate an important limitation of the 5'-nuclease DNU mechanism. FENs and other related 5'-nucleases will not be able to process damaged DNA with interstrand crosslinks surrounding the targeted phosphate diester. These products of bifunctional alkylating agents, some Michael acceptors, dialdehydes and psoralens must be repaired by an alternative nuclease that does not use unpairing of duplex 5 '-ends to control reaction specificity.

This work was supported by BBSRC grant no. BB/J00300X/1 and Marie Curie International Incoming Fellowship project no. 254386 (LDF). AB and NP thank the EPSRC and BBSRC, respectively, for studentships.

\section{Notes and references}

${ }^{a}$ Centre for Chemical Biology, Department of Chemistry, Krebs Institute, University of Sheffield, Sheffield, S3 7HF, UK. Fax: +441142229346;

Tel: +441142229478; E-mail: j.a.grasby@sheffield.ac.uk

${ }^{b}$ Current address: Institute for Glycomics, Griffith University, Gold Coast Campus, Queensland, Australia

$\dagger$ Electronic Supplementary Information (ESI) available: Full Methods, Figure S1 Substrate Schematics Figure S2 HPLC Trace of Disulfide Formation Table S1 Sequences and Mass Spectral Data of ODNs, Table S2 Substrates, See DOI: 10.1039/b000000x/

$\ddagger$ Both authors contributed equally to this work

In memory of Professor Har Gobind Khorana (1922-2011), acknowledging his legacy to the scientific community.

1 J.A. Grasby, L.D. Finger, S.E. Tsutakawa, J.M. Atack and J.A. Tainer, TIBS, 2012, 37, 74-84.

2 S.E. Tsutakawa, S. Classen, B.R. Chapados, A. Arvai, L.D. Finger, G. Guenther, C.G. Tomlinson, P. Thompson, A.H. Sarker, B. Shen, P.K. Cooper, J.A. Grasby and J.A. Tainer, Cell 2011, 145, 198-211.

3 W. Yang, J.Y. Lee and M. Nowotny, Mol. Cell, 2006, 22, 5-13.

4 K. Syson, C. Tomlinson, B.R. Chapados, J.R. Sayers, J.A. Tainer, N.H. Williams and J.A. Grasby, J. Biol. Chem., 2008, 283, 28741-28746.

5 C.G. Tomlinson, J.M. Atack, B.R. Chapados, J.A. Tainer and J.A. Grasby, Biochem. Soc. Trans., 2010, 38 433-437.

6 J. Orans, E.A. McSweeney, R.R. Iyer, M.A. Hast, H.W. Hellinga, P. Modrich and L.S. Beese, Cell, 2011, 145, 212-223.

7 L.D. Finger, S.M. Blanchard, C.A. Theimer, B. Sengerova, P. Singh, V. Chavez, F. Liu, J.A. Grasby and B. Shen, J. Biol. Chem., 2009, 284, 22184-22194.

8 T.J. Pickering, S.J. Garforth, S.J. Thorpe, J.R. Sayers and J.A. Grasby, Nucleic Acids Res., 1999, 27, 730-735.

9 J. Milton, B.A. Connolly, T.T. Nikiforov and R. Cosstick, Chem. Comm., 1993, 779-780.

10 R.S. Coleman, J.L. McCary and R.J. Perez, Tetrahedron, 1999, 55, 12009-12022.

11 I. Kuraoka, W.R. Kobertz, R.R. Ariza, M. Biggerstaff, J.M. Essigmann and R.D. Wood, J. Biol. Chem., 2000, 275, 26632-26636. 